\title{
Simple Simulated Propagation Modeling and Experimentation within and around Buildings at $2700 \mathrm{MHz}$
}

\author{
Tanay Bhatt ${ }^{1}$ and Jonathan Bredow ${ }^{2}$ \\ ${ }^{1}$ Department of Electrical Engineering, Arkansas State University, P.O. Box 1740, State University, AR 72467, USA \\ ${ }^{2}$ Department of Electrical Engineering, University of Texas at Arlington, P.O. Box 19016, Arlington, TX 76019, USA \\ Correspondence should be addressed to Tanay Bhatt; tbhatt@astate.edu
}

Received 16 September 2015; Revised 23 December 2015; Accepted 10 January 2016

Academic Editor: Sanghyun Ahn

Copyright (c) 2016 T. Bhatt and J. Bredow. This is an open access article distributed under the Creative Commons Attribution License, which permits unrestricted use, distribution, and reproduction in any medium, provided the original work is properly cited.

\begin{abstract}
There is a growing interest in understanding wave behavior in urban and suburban environment for 5th generation broadband applications. With the advent of using broadband technologies in buildings, office space and vehicle have become a necessity on a large scale. Models, predictions, and calculations for in-building, within a vehicle or near a reflective object with microscale details, are becoming highly classified in a competitive telecom environment. This paper provides an improved understanding of signal strength behavior within suburban residences with predictions prequalified using a vehicular scanner. Supporting predictions are provided by a ray tracing algorithm developed for dissertation. Results indicate signal strength variation of more than $50 \mathrm{~dB}$ from "strong signal" locations such as room centers and far corners to "weak signal" locations where shadowing and tunneling effects are evident. Based on this unique classification a scheme is proposed which indicates that specular scattering provides the major signal energy at more than $70 \%$ of the locations within the residences. Finally, an observed rake stabilizing effect is attributed to the proximity of strong scatterers.
\end{abstract}

\section{Introduction}

With the coming of new wireless technologies, Third-Generation Partnership Project (3GPP), wideband-CDMA (WCDMA), 802.11, Bluetooth, and Ultra-wide Band (UWB) systems are rapidly gaining interest and market penetration. Analysts in the USA are predicting more than 20 million broadband portable systems by the end of this decade. An increased percentage of these broadband technologies are being deployed for in-building coverage and capacity.

Indoor coverage available using radio frequencies $(1 \mathrm{GHz}-3 \mathrm{GHz})$ in the licensed UHF bands for broadband wireless systems depends on the multipath and attenuation as these electromagnetic waves pass through the structure. A formula-based approach to predicting the signal strength at any given position in the structure is desired. With the base station transmitter at an external location, this formula attempts to predict coverage or signal strength within a given structure. Initial work was presented by Walker 1983 [1] and subsequently by others [2-5]. An empirical model with transmitter located at only high altitude to study angular reflection and diffraction was presented for a rectangular building by Axiotis and Theologou [6]. An urban low elevation, lamp post levels transmitter installation study was presented with interference analysis supporting urban environment structures in $1700 \mathrm{MHz}$ [7], producing a simple path loss model with high $k$-factors at $2.5 \mathrm{GHz}$ and different building constructions [8] and using "best fit" analysis at $900 \mathrm{MHz}$ [9]. A similar study in urban microcell settings was experimented with time delayed arrivals causing higher interference through buildings [10] and a $2 \mathrm{D}$ model for ray tracing within buildings inclusive of building penetration loss [11] with some shadowing and subsequently experimental results with foliage shadowing at $5.85 \mathrm{GHz}$ [12].

An experiment in a suburban environment using time delay spread with root mean square (RMS) that doubled at every $19 \mathrm{~dB}$ of path loss was observed by Devasirvatham et al. [13]. Previous works were proposed to evaluate mobile antennas performance compared to a reference antenna with a mean effective gain in an urban/suburban environment [14] 
with multiple efficiency characterized over different polarizations; however results were outlined without a directional horn antenna to specifically quantify the angular scattering.

Specific attenuation measurements with lower sample of results, through cinder block building, was presented with linear polarization for $200 \mathrm{MHz}$ to $3 \mathrm{GHz}$ [15].

Via analysis of measurements and with simulation support, this paper focuses on the topics of improved understanding of signal strength behavior within residences in a suburban environment, in terms of features of the indoor environment; use of rake activity as an indicator of quality of the local radio environment; and impact of terrain, foliage, and nearby buildings on the indoor signal strength. A classification scheme based on rake finger activity is proposed that quantifies the impact on electromagnetic wave propagation mechanisms at various locations within a residence, that is, in terms of specular scattering, tunneling, and edge diffraction.

Once further testing is concluded, this could lead to microscale models, within residential buildings for generating a tailored indoor or on a microscale dense urban vehicular coverage prediction maps. In the second area of focus, it has been found that variability over time in the number of rake fingers used in this classification scheme based on the environment, that is, rake activity, relates to the presence of large scatterers within a few tens of wavelengths of the receiver. As long as the large scatterers do not shadow the receiver, the tendency is for these large scatterers to stabilize the number of rake fingers used compared to greater or erratic rake finger activity. In the final focus area, the degree of deviation between predictions based on commercially available RF planning tools and measured signal strengths is examined to assess effects of objects and terrain outside of the residence. In this work it is noticed that ray tracing predictions, which account only for effects of the building studied, much more closely follow measurement values for the 2 nd floor than for the 1st floor, presumably because the terrain and surrounding foliage and buildings have less impact on propagation within the 2 nd floor for a typical suburban area.

\section{Experimental Setup and Data Collection/Processing}

To enable testing in the Multichannel Multipoint Distribution Service (MMDS) frequency band of 2.5-2.7 GHz using a PCS W-CDMA scanner, a down-converter was developed. This drive test system enables the measurement of the strongest $\mathrm{N}$ signals found in each time slot of 2560 chips. The block diagram for the test setup and configuration of all equipment is shown in Figure 1. As in other CDMA systems, in WCDMA each transmitter output signal is scrambled by multiplying all of its data channels by a unique pseudonoise (PN) code, referred to in the W-CDMA specification as a scrambling code. A mobile receiver can distinguish one transmitter from another by correlating the received signal spectrum with a scrambling code that is identical to that used in the desired transmitter. Similarly, each mobile receiver output signal is scrambled with a unique scrambling code that allows the transmitter receiver to discern one mobile receiver from

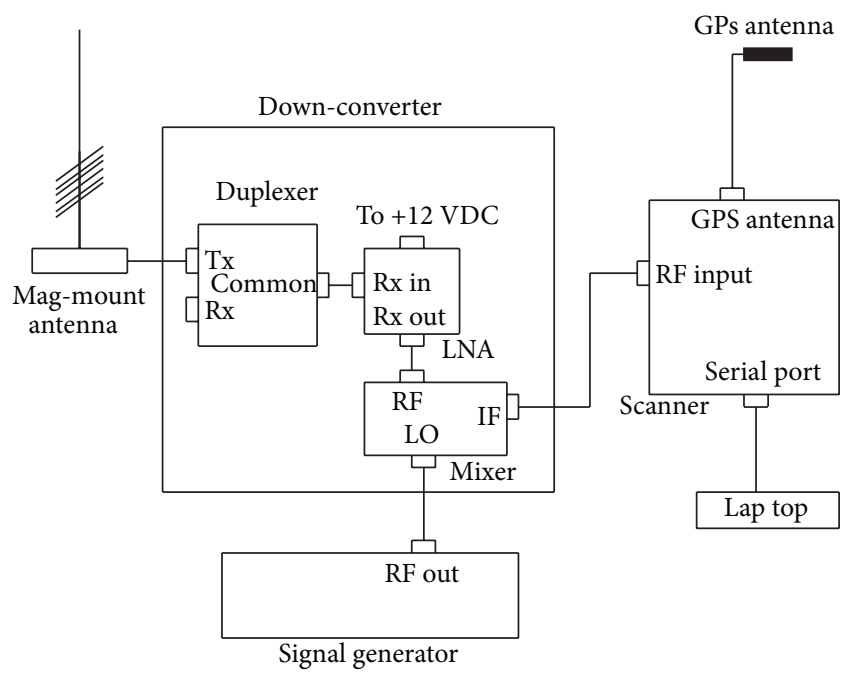

FIGURE 1: Block diagram of the portable vehicle mounted receiving system.

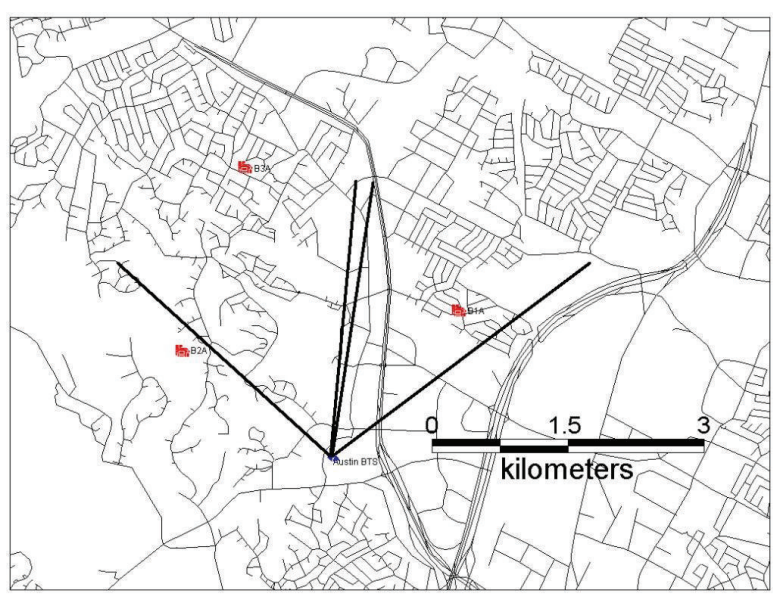

FIgURE 2: Test site locations relative to transmitter showing 65degree coverage area.

another. The scrambling codes are applied at a fixed rate of 3.840 Mcps. The purpose of the rake receiver is to perform maximal ratio combining of received multipaths in order to maximize SNR as well as despread the received chips into symbols. The 8 rake-fingers share the same time slot within the hardware prior to being processed by the combining stage.

The $2600 \mathrm{MHz}$ systems were located in Austin, TX. Transmitter antennas for the 2 sectors were located on top of a 9-story building, 39.6 meter Above Ground Level (AGL). Figure 2 shows the transmitter as well as the two residential test site locations. Power levels are estimated using the IEEE 802.16 suburban model [16].

After a thorough drive test using the portable vehicle mounted test system shown in Figure 1, the data was correlated and tuned to the predicted coverage plots for a suburban environment. The buildings were selected, as much as possible, to represent a range of natural, architectural, and local environmental characteristics, with wood framework, 
TABLE 1: Building information.

Building ID

$\mathrm{B} 2 \mathrm{~A}$

$\mathrm{B} 3 \mathrm{~A}$
Description

Building 2A: two-floor house, stone/vinyl construction, composite roof, and 2-car garage

Building 3A: single floor house, brick construction, composite sloped roof, and 2-car garage sheet rock, and composite sloped roofs. Table 1 summarizes each building construction.

To form a file of fast-fading components each sample of data collected in a given environment was normalized by the average signal strength within that environment. To control the pedestrian effects, only a single person was in the vicinity of the test gear. Collection of statistics was commenced after one minute to compensate for electromagnetic effects caused by human presence. These statistics could be correlated back to a dense urban mobile environment. The local mean was estimated by averaging the signal strength over 1000 samples; that is, the system is to collect 1 measurement at $100 \mathrm{~ms}$ intervals for 10,000 ms. Figures 3 and 4 indicate normalized receive power levels measured for various locations inside and outside of the two residential test sites. Although these tests could have directly been conducted in a dense urban vehicular settings, the decision to show comparable results in a pedestrian suburban model was taken into consideration due to logistics and personnel at hand.

Parameters considered for evaluation are mean and standard deviation of measured power, mean and standard deviation of $E_{c} / N_{0}$, RMS delay spread, and variability in rake finger activity. The primary RF design parameter for CDMA systems is the SNR or chip energy $\left(E_{c}\right)$ of a specific CDMA channel in relation to the noise $\left(N_{0}\right)$ energy, known as the $E_{c} / N_{0}$ ratio. The transmitter and receiver use an $E_{s} / N_{0}$ estimator to measure the ratio of symbol $\left(E_{s}\right)$ to noise $\left(N_{0}\right)$ energy for a particular channel (e.g., BDCH). Spread Factor (SF) is dynamically controlled within the receiver. The relationship between $E_{c} / N_{0}$ and $E_{s} / N_{0}$ is as follows:

$$
\frac{E_{c}}{N_{0}}(\mathrm{~dB})=-10 * \log _{10}(\mathrm{SF})+\frac{E_{s}}{N_{0}}
$$

Codespace can be viewed as consisting of 512 units of bandwidth. A SF $=512$ channel uses 1 unit, a SF $=256$ channel uses 2 units, a SF $=128$ channel uses 4 units, and a SF $=4$ channel uses 128 units. More units effectively correspond to a higher bandwidth channel.

The relation between bit energy $E_{b} / N_{0}$ and symbol energy $E_{s} / N_{0}$ is reasonably straightforward. For $M$-PSK/M-QAM modulation, the number of bits in each constellation symbol is

$$
k=\log _{2} M
$$

Since each symbol carries $k$ bits, the symbol to noise ration $E_{s} / N_{0}$ is $k$ times the bit to noise ratio $E_{b} / N_{0}$, or

$$
\frac{E_{s}}{N_{0}}=\frac{k E_{b}}{N_{0}} .
$$

Using the above formula, the symbol error rate versus bit energy (SNR per bit, $E_{b} / N_{0}$ ) for the system under test can thus give as

$$
P_{s, 4 \mathrm{QAM}}=\operatorname{erfc}\left(\sqrt{\frac{E_{b}}{N_{0}}}\right) .
$$

Mean and standard deviation of measured power and $E_{c} / N_{0}$ are obtained by considering measurements within a local cluster. For example, the means and standard deviations of power and $E_{c} / N_{0}$ at location 31 in Figure 4 are formed from the time average measurements at locations 27 through 31 . RMS delay spread is found according to

$$
\text { RMS delay spread }=S=\left[\frac{\sum_{k=1}^{N}\left(t_{k}-d\right)^{2} P\left(t_{k}\right)}{\sum_{k=1}^{N} P\left(t_{k}\right)}\right]^{1 / 2}
$$

where $d$ is the mean time delay, $t_{k}$ is the time delay (in seconds) relative to the start of the power delay profile (PDP), $P$ is the signal power (W), and $N$ is the index of the final PDP signal point considered. Variability in rake finger activity simply relates to how much the number of active fingers changes over the measurement interval.

\section{The Simulator}

The simulator, based on the zero-wavelength approximation treating waves as rays, has been found to give reasonable predictions of path loss and RMS delay spread for the indoor environments studied here. General ray tracing in an indoor environment assumes that direct, reflected, refracted, and diffracted rays all carry some energy to the receiver, although one or more of these may be negligible. Note that each ray is considered to be a tube that opens up as the wave propagates; this opening up represents energy spreading (the energy in the tube is considered to be constant). Although, after locating a scattering point, the reception test is performed based on reception sphere concept, this method is successfully applicable to complex environment which requires enormous computing time and memory. The reflected rays are assumed to emanate from the mirror image of the source onto the flat section of wall, ceiling, or floor, and the refracted ray is nearly parallel to the incident ray with a slight path length deviation of $\Delta d$, where

$$
\Delta d=\frac{d \sin \left(\theta_{i}-\theta_{t}\right)}{\cos \theta_{t}},
$$

$d$ is the thickness of the wall, ceiling, or floor, and $\theta_{i}, \theta_{t}$ are the angles of incidence and refraction, respectively. The diffracted field, that is, due to illumination of outside corners, is calculated using the Uniform Theory of Diffraction (UTD). 


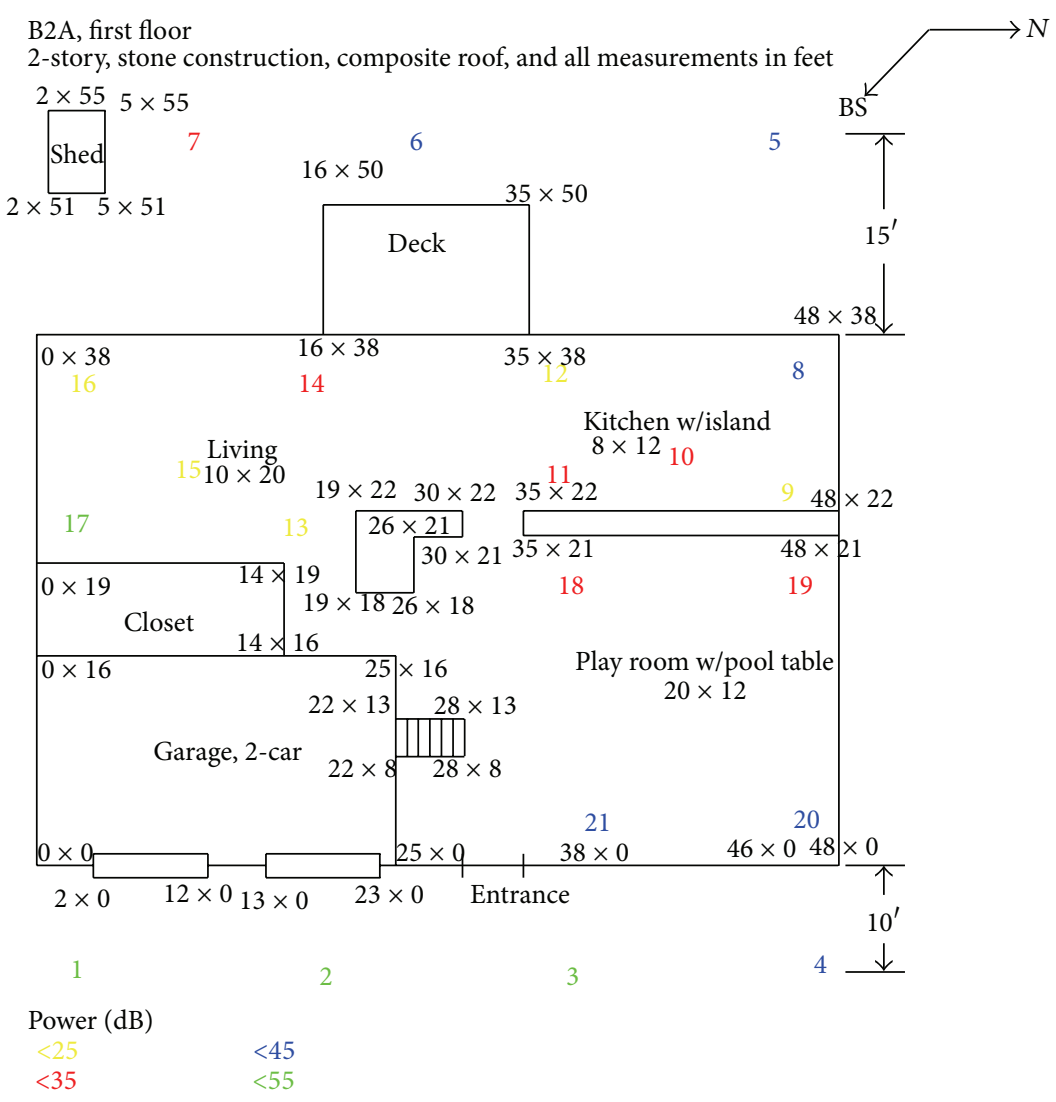

(a)

B2A, second floor

2-story, stone construction, composite roof, and all measurements in feet, $\mathrm{CPE}$ at 3-feet above ground

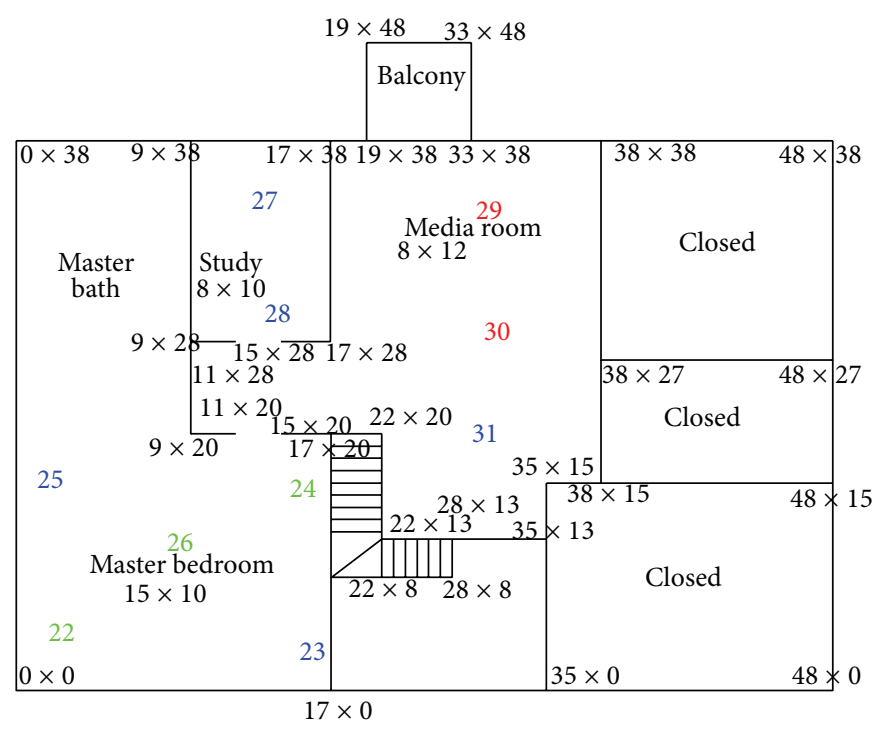

Power (dB)
$<35$
$<45$
$<55$

(b)

Figure 3: B2A Blue Print Model showing test positions and measured power for (a) first floor and (b) second floor. 
B3A, CPE at $1 \mathrm{~m}$ height at all locations Single story, brick construction, composite roof, and all measurements in feet

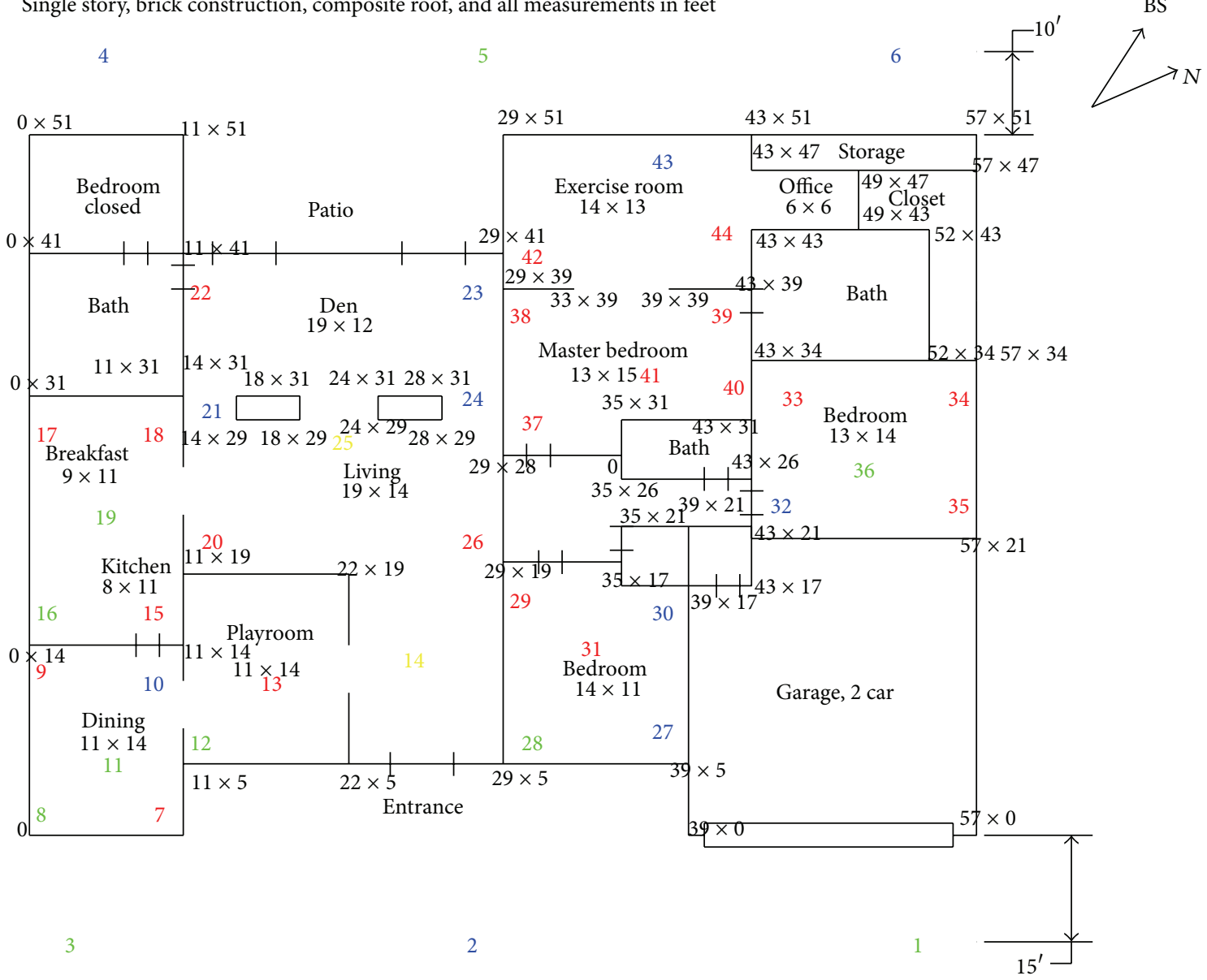

Power $(\mathrm{dB})$

Figure 4: Blue Print Model of residence B3A as tested (numbers indicate test positions and measured power).

The ray tracing-based simulator developed for this work is composed of two main parts. The first part, referred to as the ray tracer, defines the geometrical paths taken by the radio wave to reach the receiver. The second part, referred to as the electromagnetic tracer or modeler, determines the magnitude and phase of the signal along each path upon arrival at the receiver. That is, it produces a file containing information about the complex impulse response of the radio channel, that is, of the form [17]

$$
h(t)=\sum_{n=1}^{N} A_{n} \delta\left(t-\tau_{n}\right) \exp \left(-j \vartheta_{n}\right)
$$

where there are $N$ time-delayed impulses (rays), each represented by an attenuated and phase-shifted version of the original transmitted impulse for which the amplitude is $A_{n}$, the arrival time is $\tau_{n}$, and the phase is $\vartheta_{n}$. Reflected and transmitted rays are evaluated through use of geometrical optics, while diffracted rays are calculated using geometrical theory of diffraction [17]. Accordingly the complex amplitude along each path is obtained as

$$
E_{i}=E_{o} F_{t i} F_{r i}\left\{\Pi_{j} R_{j} \Pi_{k} T_{k} \Pi_{t} A_{t}\left(s^{\prime}, s\right) D_{t}\right\} \frac{e^{-j k d}}{d},
$$

where $E_{o}$ represents the reference field, $F_{t i}$ and $F_{r i}$ represent the transmitting and receiving antenna field radiation patterns in the direction of the ray, $R_{j}$ represents the reflection coefficient for the $j$ th reflection, $T_{k}$ represents the wall transmission coefficient for the $k$ th transmission, $D_{t}$ represents the diffraction coefficient for the $i$ th diffracting wedge, and $\exp (-j k d)$ represents the propagation phase factor due to the path length $d_{t}(k=2 \pi / \lambda) . A_{t}\left(s^{\prime}, s\right)$ provides the correct spatial attenuation for the diffracted rays.

Both the simulator and the floor plan module used by the simulator (the latter developed by [18]) are MATLAB ${ }^{\oplus}$ based. The source file for the floor plan module includes the material characteristics (conductivity and permittivity) of each wall, floor, and ceiling section as well as the $x, y, z$ points of each corner for the configuration. Material parameters 


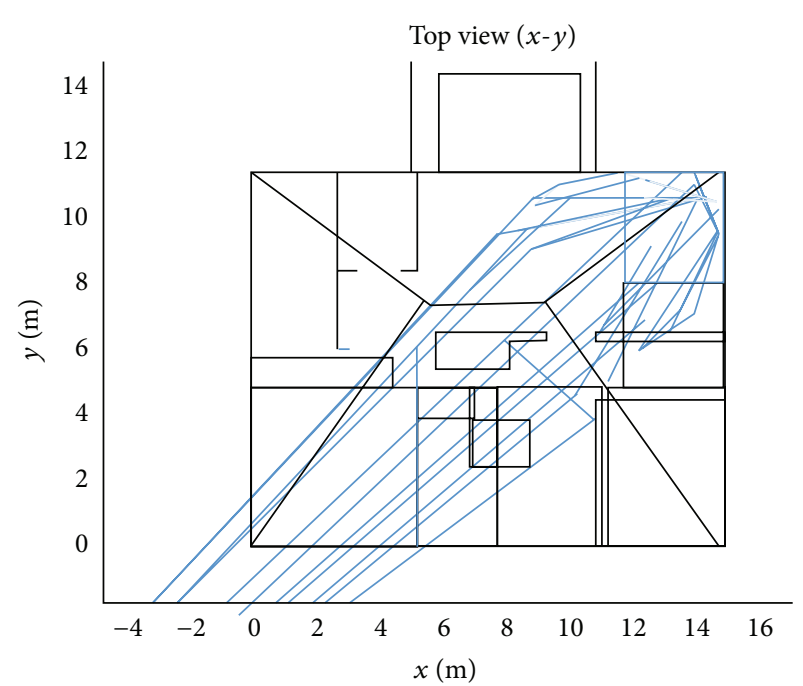

(a)

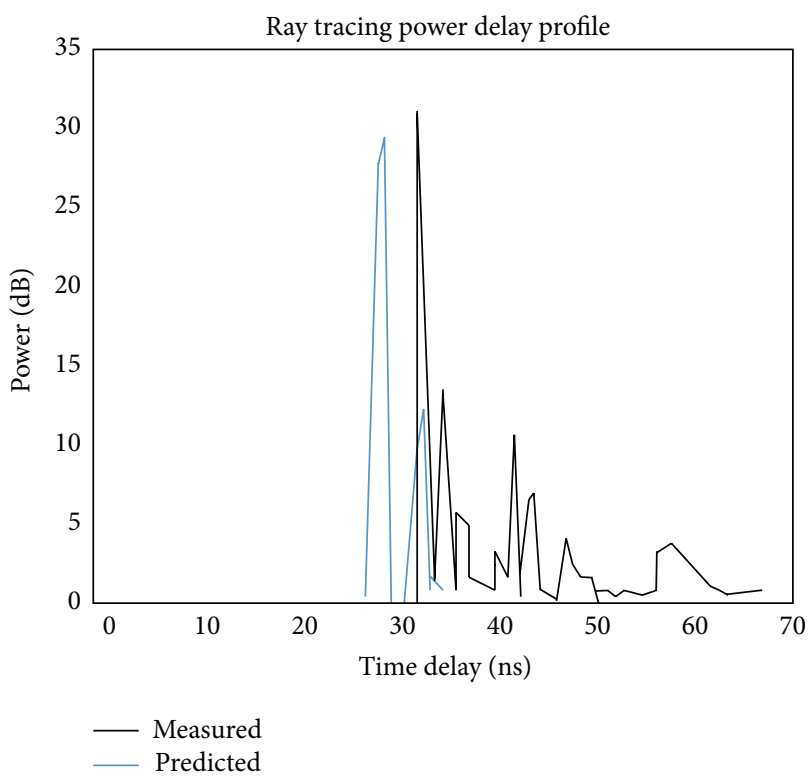

(b)

Figure 5: (a) Two-dimensional top view of location 8 of building B2A showing all contributing rays. (b) Power delay profile for location 8 building B2A.

TABle 2: Permittivity and conductivity at $2600 \mathrm{MHz}[22,23]$.

\begin{tabular}{lcc}
\hline Location & $\begin{array}{c}\text { Conductivity } \\
(\mathrm{S} / \mathrm{m})\end{array}$ & $\begin{array}{c}\text { Permittivity } \\
\text { relative }\end{array}$ \\
\hline $\begin{array}{l}\text { Ceiling (composite plus } \\
\text { wood and plaster) }\end{array}$ & 0.005 & 3 \\
$\begin{array}{l}\text { Floor (concrete) } \\
\text { Internal walls (plaster type }\end{array}$ & 0.00022 & 7 \\
of concrete) & 0.005 & 3 \\
$\begin{array}{l}\text { External walls (brick) } \\
\text { Windows }\end{array}$ & 0.005 & 3 \\
Metallic object & $1.00 E-05$ & 6 \\
& 100 & 3 \\
\hline
\end{tabular}

used for simulations are shown in Table 2, Figure 5(a) shows a sample location and contributing rays as determined by the simulator, and Figure 5(b) indicates the resulting power delay profile for that sample location.

\section{Results}

Most of the discussion concerns measured and predicted power levels and rake finger activity, as these can be readily explained in terms of features of the indoor environment. It is noticed, though, that predicted and measured RMS delay spread values matched to within $10 \mathrm{~ns}$ for the majority of locations, serving as a validation for the simulator.

From Figures 3 and 4 a consistent finding is that signal strengths at room centers are at least 10-12 $\mathrm{dB}$ higher than for the corresponding corner locations. This, of course, is only for cases where scattering, diffraction, and shadowing from objects such as cabinets and appliances do not strongly influence the signal strength. This result is expected and largely due to the narrow angular range of rays that contribute to the signal in the corner locations (as opposed to the center location). The results are similar to those realized by $[5,19]$ where $15-20 \mathrm{~dB}$ variations between corner and center locations were observed.

Figures 3 and 4 also show substantial variation of signal strength among the corners of a room. For the sites tested in this work the wave direction (from the base station) is directly onto one corner of each of the buildings. As a result a major component of energy travel is defined by a ray from corner to corner within each room. A general finding here is that the measured power is most often higher (by several $\mathrm{dB}$ ) at the far corner of the room, that is, farthest from the base station, provided that the rooms are interior rooms, that there are no exterior walls of the room that are illuminated by the base station, and that there are no strong scattering, diffraction, and shadowing effects from objects such as cabinets and appliances that may modify the pattern. This, apparently, is due to a strong corner reflector effect involving rays with lower number of reflections.

Evidence of tunneling (waveguiding) similar to Seidel and Rappaport [20], shadowing, and diffraction are clear in both of Figures 3 and 4. For example, in Figure 3, the measured signal strength at location 13 is several $\mathrm{dB}$ lower than expected due to shadowing and tunneling effects (in the short hallway-like area) and the measured signal strengths at locations 15 and 16 appear to be due to shadowing from the deck. Similarly, in Figure 4, the measured signal strengths at locations 21 and 24 are several $\mathrm{dB}$ higher than expected due to diffraction and tunneling effects involving the two pillars, whereas the signal strength at location 25 is several dB lower than expected, due to tunneling and shadowing effects inbetween the two pillars. 
TABLE 3: Summary of effects on signal strength (notice that the corresponding maxima and minima are identified with labeled arrows on the measurement curves in Figures 6 and 7).

\begin{tabular}{lc}
\hline $\begin{array}{l}\text { Location (arrow on measurement } \\
\text { curve)/noted feature(s) }\end{array}$ & Mechanism(s) \\
\hline B2A 26(G) & Room center \\
B3A 11(B), 19(D), and 36(J) & Room (far) corner \\
\hline B2A 8(A), 24(F) & Tunneling \\
B3A 8(A), 12(B), 16(C), 28(G), and 32(I) & Tunneling/scattering \\
\hline B3A 21(E)/pillars, 24(F)/pillars & Tunneling/shadowing \\
\hline B2A 11(B)/kitchen & Scattering \\
\hline B3A 25(P)/pillars & Scattering/diffraction \\
\hline B2A 14(C)/deck/shed, 15(J)/deck/kitchen, \\
and 29(K)/balcony & $\begin{array}{c}\text { Exterior wall exposed } \\
\text { to base station }\end{array}$ \\
\hline B3A 30(H)/bathroom, 39(K)/bathroom & Shadowing \\
\hline B2A 17(D), 22(E) & Shadowing/tunneling \\
B3A 43(L) & Shadowing/scattering \\
\hline B3A 20(O)/pillars & \\
\hline B3A 14(N)/pillars & \\
\hline B2A 9(H)/kitchen, 12(I)/deck, and & 13(I)/hallway-like area \\
B3A 9(M)/kitchen &
\end{tabular}

Effects of scattering and shadowing due to items in the kitchen (cabinets and large appliances) and bathroom (cabinets and bathroom fixtures) are noticeable in the measurements. For example, in Figure 3 measurements at locations 9 and 11 are several $\mathrm{dB}$ lower than expected apparently as a result of scattering and shadowing due to the kitchen items. In Figure 4, signal strength at location 9 seems to be substantially influenced (lowered) by shadowing and scattering effects from kitchen items, whereas signal strength at locations 14 and 22 are substantially lower than expected, apparently due to scattering and shadowing of bathroom items.

A summary of the mechanisms thought to be responsible for maximum and minimum signal strengths in the measurements is provided in Table 3 . Notice that the table refers to Figures 3 and 4 which show measurement locations and Figures 6 and 7 which show measured power versus location. Also shown in Figures 6 and 7 are predictions provided by the ray tracing simulator. Also note that the predicted trends are similar to those measured; however the measurements show considerably wider variations. This appears to be a result of scattering from neighboring residences as well as scattering from terrain and foliage, all of which are not considered by the simulator. Also, no knowledge about the wall composition of neighboring buildings is available. These factors can explain the disagreement between measured and simulated PDPs for large delays. Nevertheless, the delay spread and average delay show good agreement between measured and simulated power delays. One reason is due to the similarities at short delays in both plots which are of utmost importance. For the longer delays define longer delay parameters which are not in good agreement with simulated versus measured. All location results are shown in order to compare adequately the model versus measured power delay profiles. Of particular

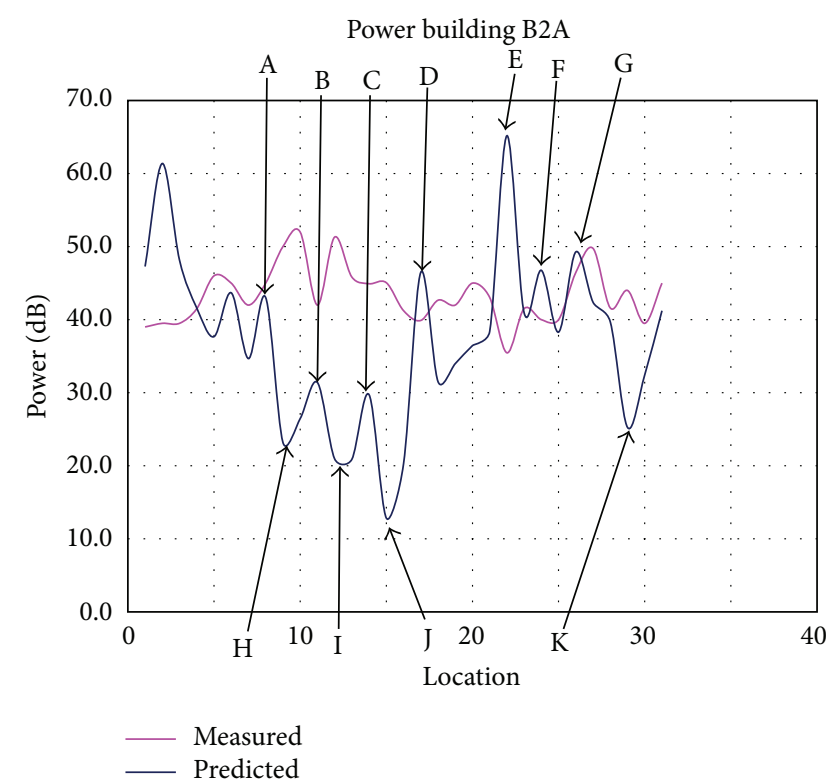

FIgURE 6: Power profile for building B2A.

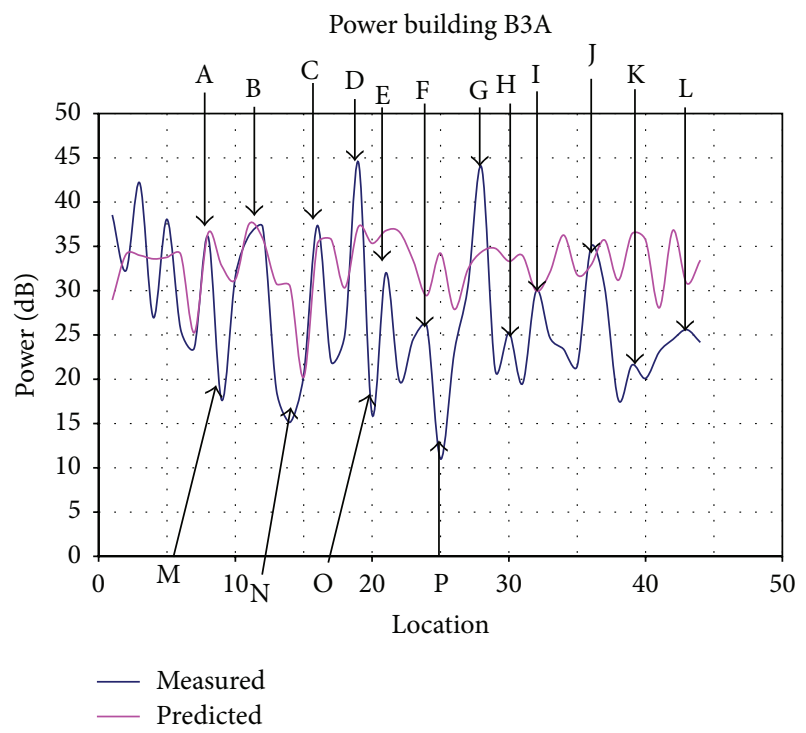

FIgURE 7: Power profile for building B3A.

note are the higher measured values on the second floor of B2A compared to those on the first floor (as expected) and the closer agreement of the simulator predictions to measurements for the second floor of $\mathrm{B} 2 \mathrm{~A}$.

A simple classification scheme was developed to determine the predominant mechanism for signal strength, as predicted by the simulator, at each of the locations in buildings $\mathrm{B} 2 \mathrm{~A}$ and $\mathrm{B} 3 \mathrm{~A}$. Classifications are specular surface scattering, including corner reflector effects, edge diffraction, and tunneling. The corner reflector effect is when a signal pulse bounces twice. Once it bounces on a vertical surface facing the receiver and then rebounces a second time from a horizontal surface towards the targeted receiver. The predominant mechanism is designated as tunneling if the 
TABLE 4: Measured rake activity according to building and location.

\begin{tabular}{lc}
\hline Building/location & $\begin{array}{c}\text { Approximate number of times active rake } \\
\text { fingers changed/second }\end{array}$ \\
\hline $\mathrm{B} 3 \mathrm{~A} / 41$ & 5 \\
$\mathrm{~B} 3 \mathrm{~A} / 10$ & 10 \\
$\mathrm{~B} 3 \mathrm{~A} / 17$ & 2 \\
$\mathrm{~B} 3 \mathrm{~A} / 24$ & 1 \\
$\mathrm{~B} 2 \mathrm{~A} / 18$ & 3 \\
$\mathrm{~B} 2 \mathrm{~A} / 10$ & 7 \\
$\mathrm{~B} 2 \mathrm{~A} / 13$ & 2.5 \\
$\mathrm{~B} 2 \mathrm{~A} / 29$ & 4 \\
\hline
\end{tabular}

majority of signal strength can be accounted for by reflected rays forming an interference pattern, similar to that observed in a waveguide, over at least a few wavelengths including the location of interest [20]. The mechanism is designated as edge diffraction if the majority of signal strength at a particular location can be accounted for by rays emanating at angles within Keller's cone from diffracting edges. The third category, that is, specular surface scattering, is assumed to be the predominant mechanism for other locations. According to this classification scheme for building B3A, signal strength at $71 \%$ of the locations was found to be primarily a result of specular scattering, edge diffraction at $14 \%$ of the locations, and tunneling at $14 \%$ of the locations. Similarly, for building B2A, signal strength at $75 \%$ of the locations was found to be primarily a result of specular scattering, edge diffraction at $13 \%$ of the locations, and tunneling at $12.5 \%$ of the locations.

\subsection{Rake Fingers Activity as a Multipath Source Indicator.} Having many rake fingers active implies that many propagation paths are present. However, given the system chip rate the paths are not resolvable unless they differ by at least about $80 \mathrm{~m}$ in length. Although spatial resolution is within 1-3 m comparative analysis when compared to ray tracing results indoor are within $8 \mathrm{~dB}$ with rake finger activity ranging from 1 to 10 ; see Table 4 . This implies that the resolvable propagation paths are normally a result of multipath propagation outside of a typical residence, that is, due to the limited building dimensions. Nonetheless, the rake finger activity or variation appears to be impacted by characteristics of the indoor channel. A study was done of the channel behavior using measurements at locations described in Figures 3 and 4, and several of these results are shown in Table 4 . The results, although with a small sample size, indicate that rake activity is lower the closer the location is to an exterior wall that faces the transmitter and lower when the location is close to but not in the shadow of strong scatterers such as pillars, cabinets, counters, and large appliances. On the other hand the level of rake activity tends to increase substantially when the location is in the shadow of large objects. In general it is assumed that if the number of rake fingers varies by more than a single finger more than 3 or 4 times over the measurement time of $10 \mathrm{~s}$, then it is considered to represent fades due to remote scatterers and not a "rake stabilizing" effect of direct paths or nearby objects such as wall corner reflections and edge diffractions, strong scattering and diffraction from cabinets, counters, and appliances. This technique is purely based on a standalone receiver compared to channel sounding which takes into account feedback information from the transmitter with a defined channel sounding spread with a weighted waveform to the subscriber station or receiver.

\section{Conclusions}

Given knowledge of the base station location, relative to a particular residence in a typical suburban area, the "strongest signal" and "weakest or most variable signal" locations can be predicted based on knowledge of the residence floor plan and locations of major scatterers such as large appliances and cabinetry. For example, room center and far corner locations were found to have the highest signal strengths; however hallway-like areas and locations near strongly diffracting objects were also found to often have higher than expected signal strengths. Although the simulator does not consider foliage and colocated building the power delay results showed comparable location specific variations when compared to the measured. Relative differences between strongest and weakest signal levels can also be estimated: for the two residences considered in this work, this difference was found to be as high as $50 \mathrm{~dB}$. Typical building characteristics can be drawn and concluded dependent on area morphology with a relatively higher sample of similar buildings and furniture adaptations within a microscale environment. With a straightforward extension of the proposed propagation mechanism classification scheme, a microscale propagation model can be developed for predicting coverage within an indoor environment. This prediction tool can also be used in a small cell dense urban environment for a mobile environment given the predefined morphological conditions. Similar hybrid environment considerations were given in previous research; see Chia [7] and Wertz et al. [21]. The correlation between in-building and mobile environment is predicted using ray tracing and measured in a similar environment with identical results [21]. As shown the indoor propagation model allows the vector-oriented definition of an arbitrary number of objects with different materials; a vehicle or building can be modeled accurately. Furthermore, for the indoor or vehicle objects a smaller discretization model is used for an urban scenario. This same discretization was considered in this paper. With a provision of slight transition requirement between suburban indoor versus dense mobile urban conditions.

Rake finger activity can be used to verify or provide additional information about important indoor propagation effects. For example, at locations exposed to exterior walls oriented toward the base station and for locations near, but not in the shadow of, large scatterers rake finger activity tends to be minimum, whereas rake activity tends to increase at locations well inside and away from large scatterers and is the highest where the location is in the shadow of large scatterers.

In our study, for the first floor of both residences, the measured signal strengths showed much higher variability than the predicted signal strengths, that is, $50 \mathrm{~dB}$ versus $20 \mathrm{~dB}$. On the other hand, for the 2 nd floor of the 2 -floor 


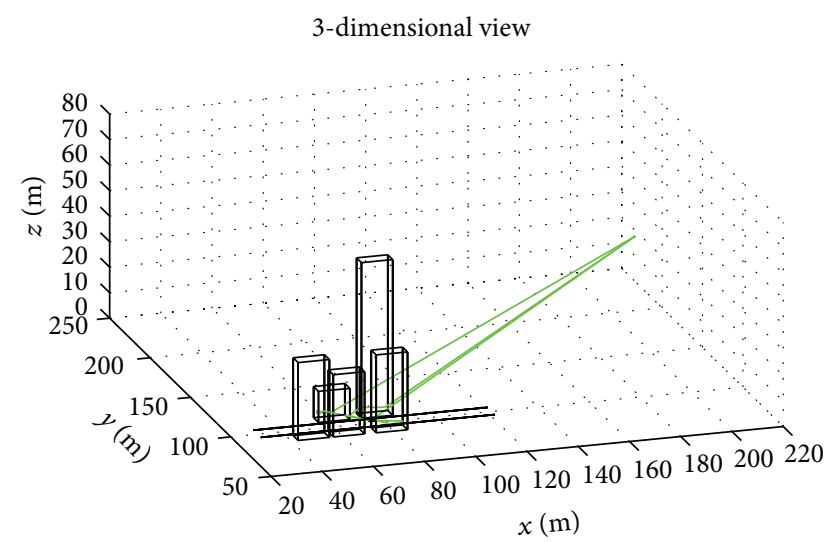

Figure 8: Vehicle configured in a dense urban clutter with ray tracing and power delay profile.

residence, the prediction trend much more closely followed the measurement trend, although still with somewhat lower variability apparently due to indoor furnishings. The inaccuracy of the building database and the lack of information about the material characteristics may cause the discrepancies founded between simulations and measured results.

Future studies to be released after receiving authorization from all parties will show similar test and measurements for a dense urban environment. Using the same ray tracing tool and subsequent measurements with a vehicle driven through a congested dense building clutter will be presented as shown in Figure 8.

\section{Conflict of Interests}

The authors declare that there is no conflict of interests regarding the publication of this paper.

\section{Acknowledgment}

The authors would like to thank Dr. Jacques Beneat, Norwich University, for the MATLAB subroutine to generate 3D buildings with unique material characteristics.

\section{References}

[1] E. H. Walker, "Penetration of radio signals into buildings in the cellular radio environment," The Bell System Technical Journal, vol. 62, no. 9, pp. 2719-2734, 1983.

[2] A. J. Motley and J. M. P. Keenan, "Personal communication radio coverage in buildings at $900 \mathrm{MHz}$ and $1700 \mathrm{MHz}$," Electronics Letters, vol. 24, no. 12, pp. 763-764, 1988.

[3] F. Lotse, J. E. Berg, and R. Bownds, "Indoor propagation measurements at $900 \mathrm{MHz}$," in Proceedings of the 42nd IEEE Vehicular Technology Conference, pp. 629-632, Denver, Colo, USA, May 1992.

[4] A. F. De Toledo, A. M. D. Turkmani, and J. Parsons, "Estimating coverage of radio transmission into and within buildings at 900 , 1800, and $2300 \mathrm{MHz}$," IEEE Personal Communications, vol. 5, no. 2 , pp. $40-47,1998$.
[5] A. F. de Toledo, Narrowband Characterization of radio transmissions into and within buildings at 900, 1800, and 2300 $\mathrm{MHz}$ [Ph.D. thesis], Department of Electrical Engineering and Electronics, University of Liverpool, Liverpool, UK, 1992.

[6] D. I. Axiotis and M. E. Theologou, "An empirical model for predicting building penetration loss at $2 \mathrm{GHz}$ for high elevation angles," IEEE Antennas and Wireless Propagation Letters, vol. 2, pp. 234-237, 2003.

[7] S. T. S. Chia, "1700 MHz urban microcells and their coverage into buildings," in Proceedings of the 7th International Conference on Antennas and Propagation (ICAP '91), pp. 504-507, York, USA, April 1991.

[8] C. Oestges and A. J. Paulraj, "Propagation into buildings for broad-band wireless access," IEEE Transactions on Vehicular Technology, vol. 53, no. 2, pp. 521-526, 2004.

[9] D. Peña, R. Feick, H. D. Hristov, and W. Grote, "Measurement and modeling of propagation losses in brick and concrete walls for the 900-MHz band," IEEE Transactions on Antennas and Propagation, vol. 51, no. 1, pp. 31-39, 2003.

[10] Y. L. C. de Jong, M. H. A. J. Herben, J.-F. Wagen, and A. Mawira, "Transmission of UHF radiowaves through buildings in urban microcell environments," Electronics Letters, vol. 35, no. 9, pp. 743-745, 1999.

[11] Y. L. C. de Jong, M. H. J. L. Koelen, and M. H. A. J. Herben, "A building-transmission model for improved propagation prediction in urban microcells," IEEE Transactions on Vehicular Technology, vol. 53, no. 2, pp. 490-502, 2004.

[12] G. Durgin, T. S. Rappaport, and H. Xu, " $5.85-\mathrm{GHz}$ radio path loss and penetration loss measurements in and around homes and trees," IEEE Communications Letters, vol. 2, no. 3, pp. 70-72, 1998.

[13] D. M. J. Devasirvatham, R. R. Murray, and D. R. Wolter, "Time delay spread measurements in a wireless local loop test bed," in Proceedings of the IEEE 45th Vehicular Technology Conference, pp. 241-245, July 1995.

[14] I. Z. Kovacs, P. C. F. Eggers, and K. Olesen, "Comparison of mean effective gains of different DCS 1800 handset antennas in urban and suburban environments," in Proceedings of the 48th IEEE Vehicular Technology Conference (VTC '98), vol. 3, pp. 1974-1978, IEEE, Ottawa, Canada, May 1998.

[15] C. D. Taylor, S. J. Gutierrez, S. L. Langdon, and K. L. Murphy, "On the propagation of RF into a building constructed of cinder block over the frequency range $200 \mathrm{MHz}$ to $3 \mathrm{GHz}$," IEEE Transactions on Electromagnetic Compatibility, vol. 41, no. 1, pp. 4649, 1999.

[16] V. Erceg, K. V. S. Hari, M. S. Smith et al., "Channel models for fixed wireless applications," Contribution IEEE 802.16.3c01/29r1, 2001.

[17] J. B. Keller, "Geometrical theory of diffraction," Journal of the Optical Society of America, vol. 52, pp. 116-130, 1962.

[18] N. Bailey and N. Deslandes, Senior Project Report-Secure Wireless Communication Deployment Tool, Norwich University, 2003, Director J. Beneat.

[19] G. E. Athanasiadou and A. R. Nix, "A novel 3-d indoor raytracing propagation model: the path generator and evaluation of narrow-band and wide-band predictions," IEEE Transactions on Vehicular Technology, vol. 49, no. 4, pp. 1152-1168, 2000.

[20] S. Y. Seidel and T. S. Rappaport, "Site-specific propagation prediction for wireless in-building personal communication system design," IEEE Transactions on Vehicular Technology, vol. 43, no. 4, pp. 879-891, 1994. 
[21] P. Wertz, D. Zimmermann, F. M. Landstorfer, G. Wölfle, and R. Hoppe, "Hybrid ray optical models for the penetration of radio waves into enclosed spaces," in Proceedings of the IEEE 58th Vehicular Technology Conference (VTC '03), vol. 1, pp. 109113, IEEE, October 2003.

[22] A. R. Von Hippel, Dielectric Materials and Applications, John Wiley \& Sons, New York, NY, USA, 1954.

[23] American Institute of Physics Handbook, McGraw-Hill, New York, NY, USA, 3rd edition, 1972. 


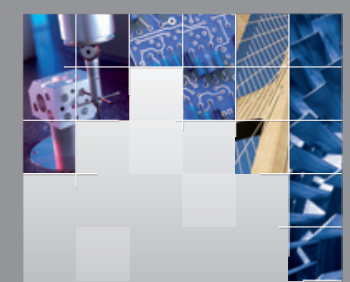

\section{Enfincering}
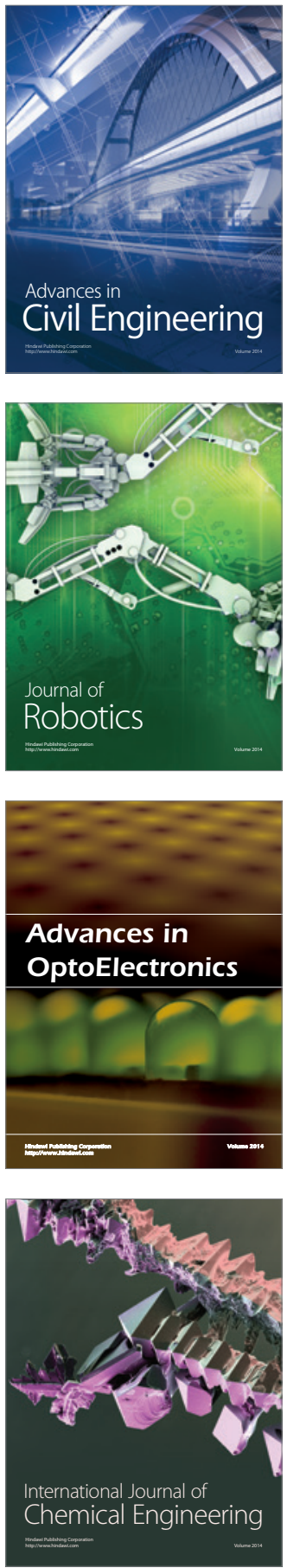

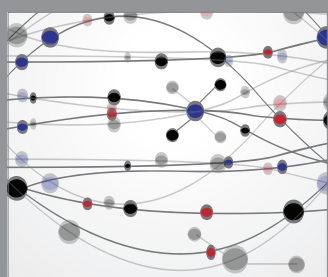

The Scientific World Journal

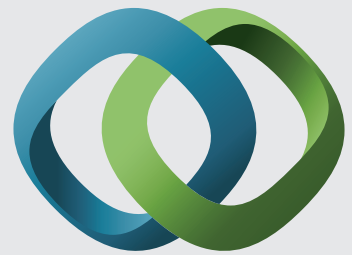

\section{Hindawi}

Submit your manuscripts at

http://www.hindawi.com
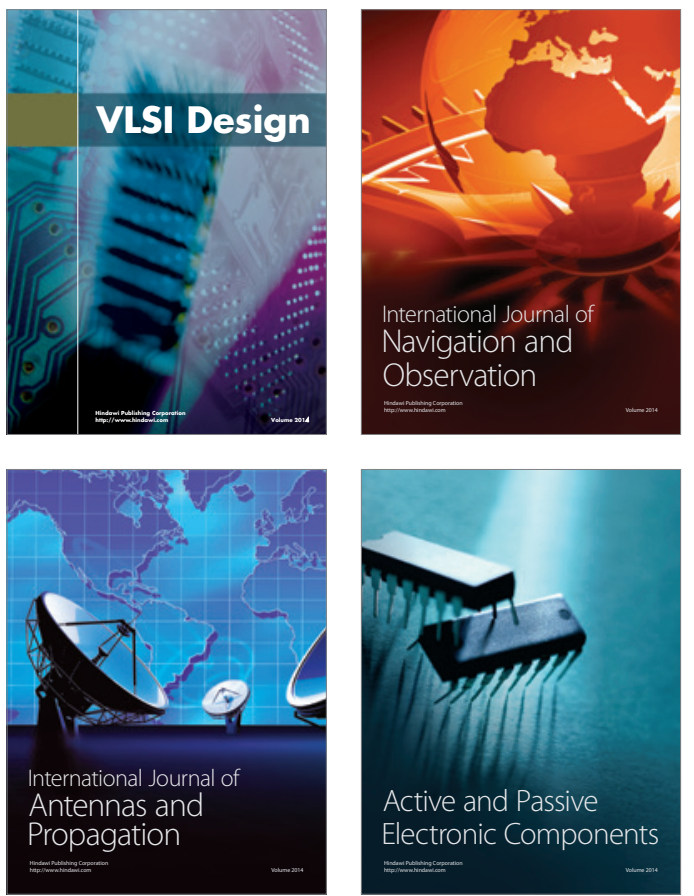
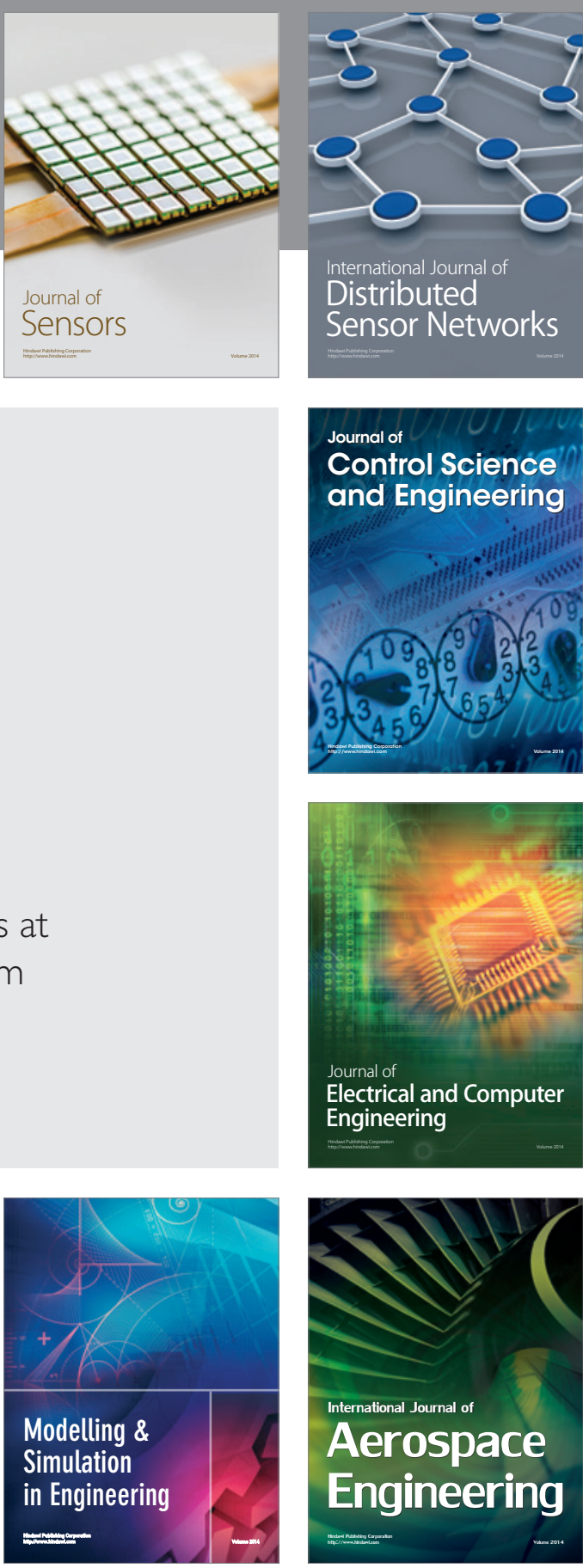

International Journal of

Distributed

Sensor Networks

Journal of

Control Science

and Engineering
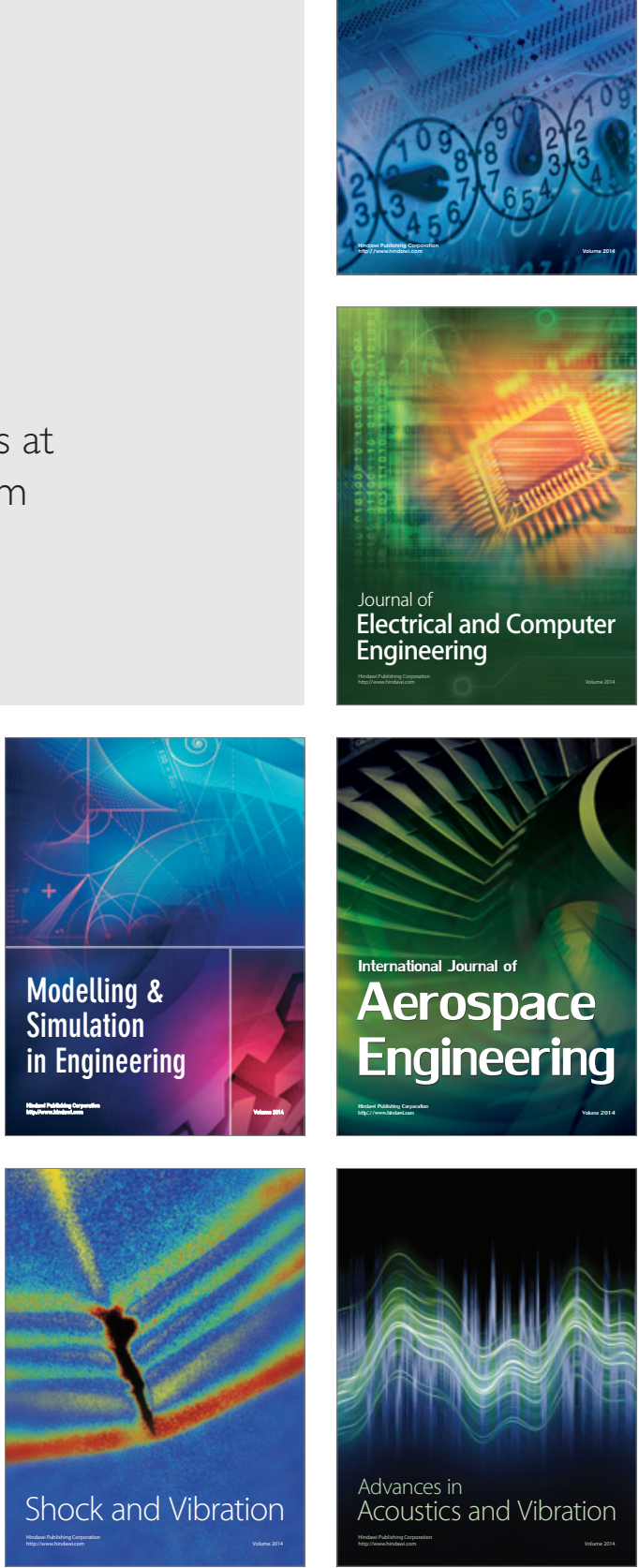